\title{
Outpatient versus Inpatient Observation after Percutaneous Native Kidney Biopsy: A Cost Minimization Study
}

\author{
Saugar Maripuri a David F. Penson ${ }^{\text {b, c }}$ T. Alp Ikizler ${ }^{a}$ Kerri L. Cavanaugh ${ }^{a}$ \\ a Division of Nephrology and Hypertension, ${ }^{b}$ Department of Urologic Surgery, at Vanderbilt University Medical \\ Center and ' VA Tennessee Valley Geriatric Research, Education, and Clinical Center (GRECC), Nashville, Tenn., USA
}

\section{Key Words}

Kidney biopsy $\cdot$ Decision analysis $\cdot$ Institutional costs

\begin{abstract}
Background/Aims: Percutaneous kidney biopsy (PKB) is the primary diagnostic tool for kidney disease. Outpatient 'day surgery' (ODS) following PKB in low-risk patients has previously been described as a safe alternative to inpatient observation (IO). This study aims to determine if ODS is less costly compared to 10 while accounting for all institutional costs (IC) associated with post-PKB complications, including death. Methods: A cost minimization study was performed using decision analysis methodology which models relative costs in relation to outcome probabilities yielding an optimum decision. The potential outcomes included major complications (bleeding requiring blood transfusion or advanced intervention), minor complications (bleeding or pain requiring additional observation), and death. Probabilities were obtained from the published literature and a base case was selected. IC were obtained for all complications from institutional activity-based cost estimates. The base case assumed a complication rate of $10 \%$ with major bleeding occurring in $2.5 \%$ of patients (for both arms) and death in 0.1 and $0.15 \%$ of IO and ODS patients, respectively. Results: ODS costs USD 1,394 per biopsy compared to USD 1,800 for IO inclusive
\end{abstract}

of all complications. IC for ODS remain less when overall complications $<20 \%$, major complications $<5.5 \%$, and IC per death <USD 1.125 million. ODS remained favored through sensitivity analysis. Conclusion: Outpatient management after PKB for low-risk patients costs less from the institutional perspective compared to $\mathrm{IO}$, inclusive of complications and death. ODS should be considered for low-risk patients undergoing native kidney biopsy.

Copyright $\odot 2011$ S. Karger AG, Basel

\section{Introduction}

Percutaneous biopsy of native kidneys is an important diagnostic tool for clinicians seeking a diagnosis for patients with kidney disease. The primary risks for percutaneous kidney biopsy (PKB) range from mild complications such as post-procedural pain and gross hematuria to major complications such as large hematomas requiring blood transfusion, uncontrolled bleeding requiring embolization or surgical nephrectomy, and rarely death [1]. The technique for obtaining tissue has evolved with the emergence of direct ultrasound guidance as the standard of care, dramatically improving procedural safety and diagnostic yield [2].

\section{KARGER}

두 2011 S. Karger AG, Basel

Fax +41613061234 E-Mail karger@karger.ch www.karger.com www.karger.com/ajn
Saugar Maripuri, MD

Division of Nephrology and Hypertension, Vanderbilt University Medical Center

1161 21st Avenue MCN S-3223

Nashville, TN 37232 (USA)

Tel. +1 615343 6104, E-Mail saugar.maripuri@vanderbilt.edu 
While a number of centers in the US require overnight inpatient observation (IO) following PKB, several studies have demonstrated the safety of the outpatient 'day surgery' (ODS) approach [3-9]. This strategy discharges patients within 4-6 h after biopsy if their course was uncomplicated and lacked risk factors for bleeding, such as uncontrolled hypertension, use of anticoagulants, or underlying advanced chronic kidney disease. Complications were rare with no deaths reported in the published literature.

Rising healthcare costs and increased use of capitated payments have raised awareness for strategies that reduce institutional expense [10]. From this economic perspective, the outpatient strategy should be associated with lower immediate costs to the institution. However, little is known how the long-term costs of subsequent readmission, major complications, or death would impact the total institutional cost (IC) per biopsy - which may eliminate any cost savings.

This cost minimization study utilizes decision analysis to determine the least costly strategy for managing patients after $\mathrm{PKB}$ inclusive of all potential IC, including complications and death.

\section{Materials and Methods}

Decision analysis is an analytic technique that is used to examine the relative costs, effectiveness, and cost-effectiveness of alternative diagnostic or therapeutic strategies [11]. Components of decision analysis include: defining the problem, determining an alternative strategy, describing the potential clinical outcomes from both strategies, estimating probabilities of each outcome along with potential costs and utilities, and calculating the expected outcome to determine the optimal decision. This study utilized these techniques in the form of cost minimization, which attempts to determine the least costly strategy while factoring in the costs of all potential outcomes, including major complications and death. This study did not examine the relative utility (effectiveness) of each outcome, other than survival or death, as longterm comorbid states after a PKB complication would be difficult to distinguish from the loss of kidney function due to the native disease process.

\section{Selection of the Base Case}

A base case was determined by examination of the literature. The studies that examined the safety of PKB in an outpatient generally selected patients of lower risk profiles $[4,5]$. Therefore, the defined population for this study included a hypothetical cohort of patients with no known risk factors for bleeding (i.e. thrombocytopenia, clotting disorders, use of anticoagulants or need for bridging anticoagulants), systolic blood pressure of $<160 \mathrm{~mm} \mathrm{Hg}$ at time of biopsy, absence of advanced chronic kidney disease (stage IV and V), and no evidence of bleeding on immediate postbiopsy ultrasonography. All patients regardless of the decision to discharge home or observe overnight are assumed to have been monitored after PKB for at least $4 \mathrm{~h}$ with no evidence of hemodynamic instability, postprocedural pain, gross hematuria, or drop in hematocrit. The perspective was that of the hospital/payer and indirect costs such as time off work and outpatient medications, which are not usually borne by the payer, were not included.

Model for Decision Analysis

Figure 1 is a graphical representation of the decision tree model. It represents a straightforward approach to the potential outcomes after PKB for both ODS and IO arms. It assumes that potential complications will occur within the first $23 \mathrm{~h}$ after PKB. The ODS arm assumes that most patients with a complication will return to an emergency department for evaluation and includes the potential of death prior to re-admission to the hospital.

Minor bleeding was defined as postprocedure pain, gross hematuria, or small hematomas seen on repeat imaging not requiring blood transfusion. Major bleeding was defined as an event requiring blood transfusion and/or endovascular embolization by interventional radiology to halt a bleeding vessel. Surgical nephrectomy was not included in the model to limit model complexity and since the costs and risks of the procedure would be shared equally between both arms, making it unlikely to affect the primary outcome or sensitivity analysis.

\section{Estimation of Probabilities}

Relative probabilities of adverse events after PKB were obtained from the literature $[1,12-16]$ and are summarized in table 1. A base probability for each outcome was selected that erred on the side of overestimating the impact of major complications, including death. Probabilities for the ODS arm of the decision tree were assumed similar to that of inpatients, despite evidence in the literature that very few major complications occurred among outpatients. This assumption was made to account for publication bias, as less favorable outcomes among outpatients would be less likely to be published. The outpatient arm required an emergency department visit for most complications and included the possibility of delayed diagnosis and death prior to readmission. The overall risk of death after PKB among outpatients was modeled at $0.15 \%$, while inpatients were modeled at $0.1 \%$. This differential in risk of death was chosen a priori to account for the risk of delayed diagnosis of late bleeds, which may account for $1 / 3$ of all complications [16], and the possibility of death prior to hospital admission among outpatients.

\section{Estimation of Institutional Costs}

The IC of each outcome was obtained from activity-based cost estimates as determined by the Vanderbilt University Medical Center Finance Department and is summarized in table 2. A major complication with intervention assumed 2 ICU days, 2 floor days, 4 units of packed red cell transfusion, and endovascular embolization by interventional radiology. A major complication with only transfusion assumed one ICU day, one floor day, and 2 units of packed red cell transfusion. A minor complication assumed one additional floor day. Any complication, minor or major, was felt to warrant a CT scan of the abdomen/pelvis and was included in the cost analysis. Additional cost was incurred for outpatients due to the utilization of the emergency department, which included imaging by CT scan and laboratory studies. ICs reflect actual costs to the institution, and do not include physician charges. 


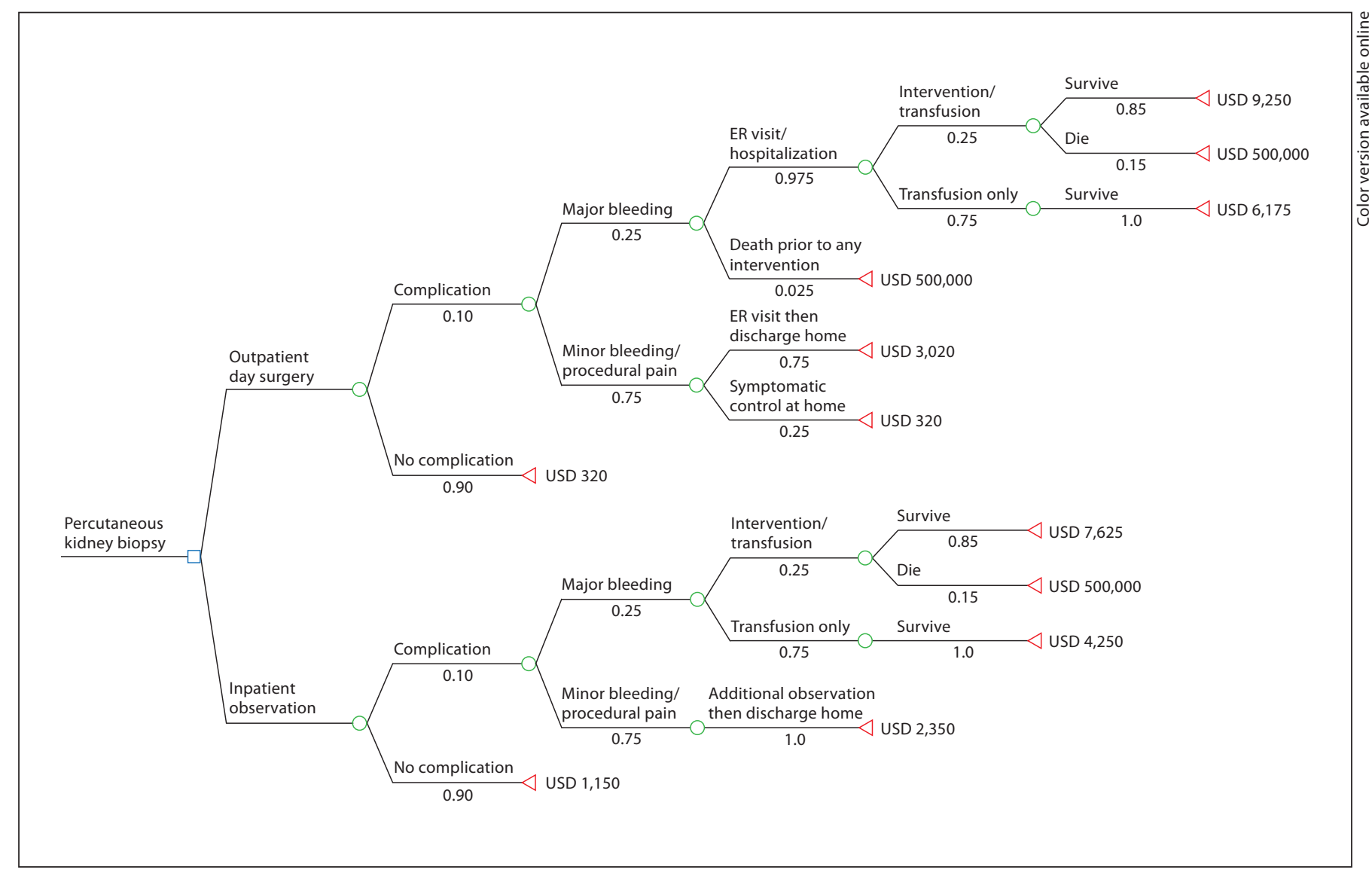

Fig. 1. Decision analysis model comparing ODS (top arm) to IO (bottom arm). The square represents a decision node; a circle represents a chance node (a probability of two events occurring); a triangle represents an end point node. Costs for each outcome are estimated from institutional finance calculations (see 'Materials and Methods').

Table 1. Probability of complications after PKB

\begin{tabular}{llll}
\hline Variable probability & $\begin{array}{l}\text { Modeled } \\
\text { probability }\end{array}$ & $\begin{array}{l}\text { Range in } \\
\text { literature }\end{array}$ & References \\
\hline Any complication & 0.1 & $0.05-0.31$ & {$[1,12,14-16]$} \\
Minor bleeding event & 0.075 & $0.067-0.24$ & {$[14-16]$} \\
Major bleeding event & 0.025 & $0.01-0.08$ & {$[1,12-16]$} \\
Transfusion & 0.025 & $0-0.07$ & {$[13,15,16]$} \\
Advanced intervention & 0.006 & $0.004-0.012$ & {$[12-16]$} \\
Death & 0.001 & $0-0.002$ & {$[1,12-16]$} \\
\hline
\end{tabular}

Modeled probabilities were selected from the range in the literature assuming a low-risk patient undergoing PKB.
Table 2. Summary of estimated ICs

\begin{tabular}{|c|c|}
\hline Expense & $\begin{array}{l}\text { Estimated } \\
\text { cost, USD }\end{array}$ \\
\hline Ultrasound-guided kidney biopsy ${ }^{1}$ & 162.00 \\
\hline Overnight observation, $23 \mathrm{~h}^{2}$ & 897.00 \\
\hline Non-monitored medical bed, per day ${ }^{2}$ & 931.00 \\
\hline ICU medical bed with monitoring, per day ${ }^{2}$ & $1,611.00$ \\
\hline Transfusion of packed red cells, per unit & 152.50 \\
\hline CT scan of the abdomen and pelvis & 251.00 \\
\hline Endovascular embolization by interventional radiology ${ }^{1}$ & 531.00 \\
\hline Emergency department visit & \\
\hline (including labs, CT scan, nursing care) & $2,678.00$ \\
\hline
\end{tabular}

\footnotetext{
${ }^{1}$ Includes costs associated with medical imaging, radiology technician, and supplies.

${ }^{2}$ Includes costs associated with nursing care.
} 
Table 3. One-way sensitivity analyses for PKB complications and institutional cost

\begin{tabular}{lcc}
\hline Outcome & Range tested & Range where ODS is favored \\
\hline Overall complication rate, \% & $0-50$ & $<19.6$ \\
Major bleeding event rate (among all patients with a complication), \% & $0-100$ & $<54.7$ \\
Advanced intervention rate (among all patients with a major complication), \% & $25-75$ & ODS favored throughout range \\
Uncomplicated ER visit and discharge (among outpatients with a minor complication at home), \% & $0-100$ & ODS favored throughout range \\
Death prior to re-hospitalization (among outpatients with a major complication at home), \% & $0-25$ & $<5.9$ \\
Institutional cost of death, USD millions & $0.5-2.0$ & $<1.175$ \\
\hline
\end{tabular}

The cost of a death from kidney biopsy was estimated as the potential liability to the institution, such as increased medical malpractice premiums, legal costs, and potential settlement from a risk management perspective and is not an estimate of the value of a patient's life.

\section{Sensitivity Analyses}

Sensitivity analysis was performed on each variable in the model to determine the threshold probability or cost yielding a change in decision. This type of analysis was also utilized to explore the possibility of a differential cost per death, as a 'day surgery' patient suffering a serious or fatal outcome shortly after discharge may be more likely to engage in litigation and therefore increase the potential cost to the institution. This was achieved through the use of a cost multiplier, which was manipulated through sensitivity analysis. All modeling and analysis was performed using the TreeAge decision analysis package, version 2011.

\section{Results}

Assuming the base case and model as presented, the average cost per PKB accounting for all complications, including death, was USD 1,394 for the outpatient arm compared to USD 1,800 for the inpatient arm, a potential savings of USD 406 per biopsy (22.5\%).

Sensitivity analyses show the thresholds for where ODS would continue to be favored and is summarized in table 3. ODS remains the favored strategy as long as the overall complication rate is less than $19.6 \%$ and if major bleeding accounts for less than $54.7 \%$ of all complications (less than $5.5 \%$ of all biopsies overall). The IC per death would need to exceed USD 1.175 million for IO to be favored. For outpatients encountering a major bleeding event while at home, death prior to hospitalization would need to exceed $5.9 \%$ of cases to favor IO.

The model and base case assumed the ICs per death for both inpatients and outpatients are equal, which may not be accurate. To examine this possibility, the differential cost of death was analyzed using a cost multiplier. Sensitivity analysis showed that IO would be favored only if the IC per death for an outpatient exceeded 1.53 times that of an inpatient, assuming the base cost of USD 500,000 . Figure 2 demonstrates this effect for a variety of different base ICs per death; as the base cost decreases, the cost differential must substantially increase to continue favoring IO.

Another clinical situation that should be considered is the possibility of outpatients requiring a major intervention at a much higher rate due to delayed diagnosis. Figure 3 demonstrates that the outpatient strategy remains favored as long as the risk of an advanced intervention among outpatients presenting to the ER with a major bleeding event remains less than $46.7 \%$.

\section{Discussion}

The results of our study indicate that outpatient 'day surgery' after PKB may have lower institutional costs inclusive of all potential complications compared to inpatient observation for low-risk patients. Sensitivity analysis demonstrated that this relationship remained, even at high probabilities of complications and costs. Outpatient management after PKB can be considered in patients with low risk for bleeding, including lack of known risk factors, absence of advanced chronic kidney disease, normal post-procedure ultrasonography, and hemodynamic stability in the immediate hours following PKB.

Outpatient management after $\mathrm{PKB}$ is a safe alternative in low-risk patients [3-9]. Despite this, routine use of the outpatient approach remains dependent on the practicing clinician. Proponents cite the institutional cost benefits, low complication rates, and the flexibility of risk stratification. It also allows patients to avoid hospitalization and recover at home. In addition, immediate [17] and/or delayed (1-2 h) postbiopsy ultrasound [15] could predict bleeding risk, providing additional reassurance prior to discharge. The latter was not modeled in this analysis, but the incremental cost of a limited renal ultrasound would fall well under the incremental cost difference observed in 


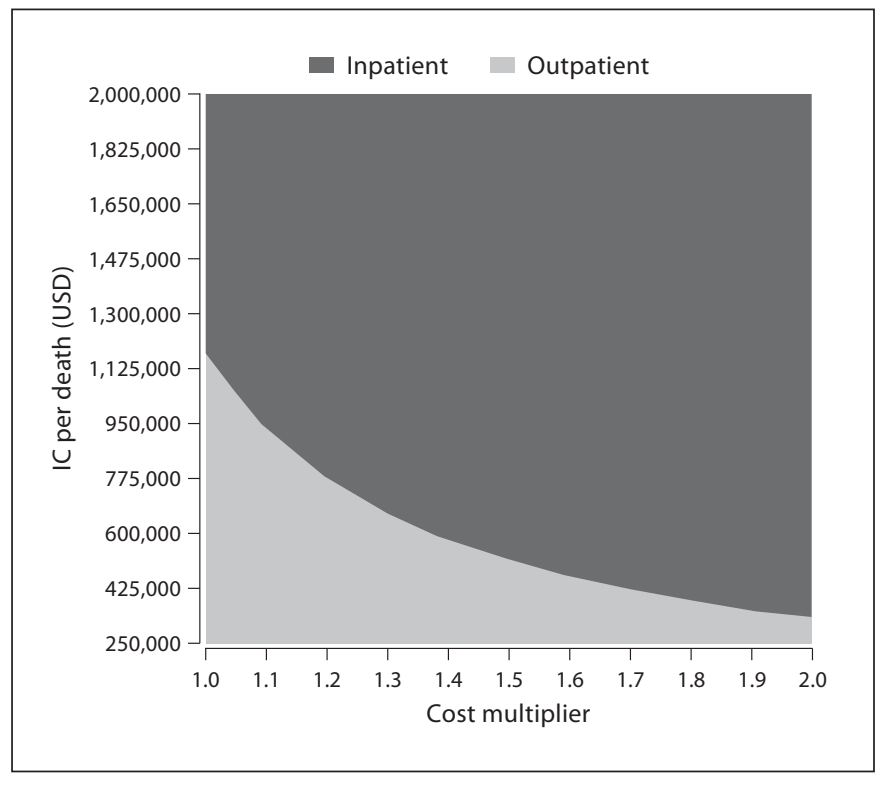

Fig. 2. Two-way sensitivity analysis for the effect of differential IC of death between outpatients and inpatients. The respective shaded areas represent the levels where each management style is favored for different degrees of differential cost (estimated through the cost multiplier) and base IC of death.

this study. Critics note that while a significant portion of complications will be detected early, there remain a large proportion of adverse events, perhaps as high as one third $[16,18]$, that are missed after discharge home. However, the vast majority of complications that occur after $12 \mathrm{~h}$ are minor with most major complications (89\%) occurring prior to this [16].

While the published literature supports the safety of outpatient management after PKB, it is important to recognize that this strategy still bears risk. While the observed risk of complications is low with no deaths reported in the literature among outpatients, it is unrealistic to conclude that ODS is safer than IO. The lower risk of complications and death may stem from the selection of lower risk patients or publication bias. This analysis postulated that the theoretical risk of death among outpatients would be higher than among inpatients, $0.15 \%$ compared to $0.10 \%$. This assumption was based on the observation that delayed bleeds do occur [16] and outpatients may be less likely to promptly recognize the symptoms of a major complication. It also raised the required threshold needed to reject the null hypothesis that IO was less costly inclusive of all complications. Given that this analysis ultimately favored outpatient management, this

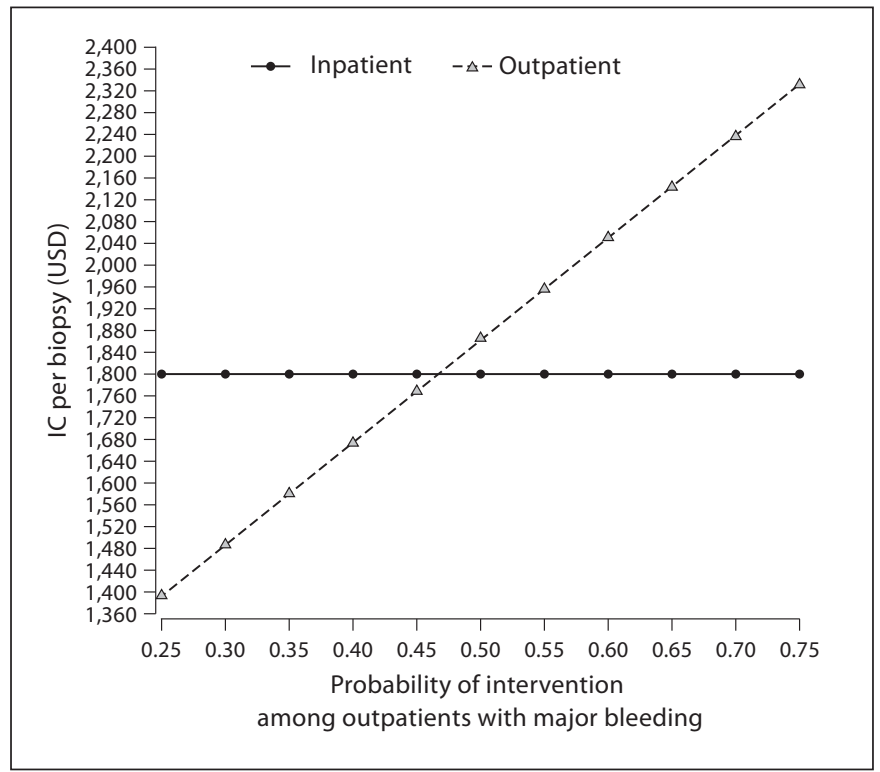

Fig. 3. One-way sensitivity analysis for a differential probability in advanced intervention among outpatients with major bleeding. Outpatient management remains the favored strategy if the risk of a major intervention among outpatients presenting with a major bleed does not exceed 0.467 (46.7\%).

further strengthens our conclusion that ODS is less costly from the institutional perspective.

Despite the observed safety of outpatient PKB inclusive of these risks of delayed bleeds, some clinicians may prefer the perceived safety of $\mathrm{IO}$, and this preference may remain despite knowledge of the considerable costs associated with this policy. However, it is important to recognize that the strategy of IO does not prevent deaths from occurring, bleeding events can occur after $23 \mathrm{~h}$ [16], and delayed diagnosis can occur even for inpatients. This is why IO is an extremely expensive strategy if the goal was only to prevent patient death. The overall risk of death as presented in this study is 1 per 1,000 and 1.5 per 1,000 for inpatients and outpatients, respectively. Assuming an incremental cost of USD 406 per biopsy for IO inclusive of all complications, the incremental cost for preventing one death through IO exceeds USD 800,000. This may appear to be worth the expense at first glance but it exceeds the base estimate for cost per death by at least USD 300,000. While this may seem reasonable to a clinician who is risk adverse, it constitutes a considerable expense to the institution given PKB has known and unavoidable risks even in the best of monitoring environments. It is also important to acknowledge that despite all the evidence presented in 
this analysis, some clinicians will remain steadfast in the belief that even a slightly increased risk of death associated with the outpatient strategy would be unacceptable, regardless of the cost savings. The model does not address this ethical question that will come under even greater scrutiny in the near future as we continue to address rising costs in health care.

Assuming one is willing to accept the slightly increased risk of death associated with the outpatient perspective, it is also important to recognize that this analysis is from the institutional perspective. The quantification of cost is therefore based on actual institutional estimates from one center. The IC of an unexpected patient death is very difficult to quantify and can be easily confused with the societal cost of death, which primarily quantifies lost productivity such as wages. It would be inappropriate to estimate societal cost for this analysis as it does not reflect a direct cost to the institution. Similarly, a cost-effectiveness analysis was not performed in this analysis as the majority of costs of the disutility (effectiveness) of a major complication, such as time off work and lost wages, are borne by society and not the institution or payer. An estimate was therefore determined to reflect the IC associated with risk management, increased insurance premiums, and litigation costs. Since these costs can greatly differ at varying institutions, sensitivity analysis is the best way to interpret the impact of the cost of death on the overall cost per biopsy. Despite our belief that the base cost of death is overestimated in this analysis, outpatient management was still favored even if that cost doubled.

This analysis does not address nor does it support the use of ODS for all patients undergoing PKB. The base case specifically considered patients of low-risk profiles and the results and conclusions of this analysis should not be applied to patients with risk factors for a bleeding event. In fact, our findings also suggest that patients with specific risk factors that raise concern for a complication should be observed overnight, especially if the perceived risk for a major complication in an individual patient exceeds 5\% (as determined by sensitivity analysis).

This analysis assumed that patients observed through an ODS approach have reliable access to emergency medical care in the event a complication were to occur. For patients who live in outlying or rural areas, or who do not have reliable emergency services in their area of residence, ODS may not be the preferred strategy as the risk of delayed recognition or death may exceed the cost benefit achieved from outpatient management.
This study has several limitations. First, our model is a generalization of a potentially complex hospital course. Each outcome follows a standardized course and does not attempt to analyze real patient encounters or actual cost data, as the latter would be subject to significant cost variation based on provider management style. This limitation, however, is balanced by the strength gained in sensitivity analysis and ability to thoroughly test each assumption. Second, the probability of complications utilized in this model is an estimate based on the available literature and may vary depending on the institution, procedural skill, use of direct ultrasonography, number of cores obtained, and other factors. All costs reported are specific to our institution but could be generalizable to other institutions of similar size in the US. Lastly, the IC of a patient death is very difficult to quantify. Not all patients encountering serious or lethal adverse events choose to pursue litigation nor are most adverse events associated with $\mathrm{PKB}$ due to gross negligence or medical malpractice. Therefore, the estimates for IC per death may be overestimated in this study.

\section{Conclusion}

Outpatient 'day surgery' after PKB, a medically safe alternative to inpatient observation for low-risk patients, appears to cost less from the institutional perspective. The cost advantage for outpatient management persists through sensitivity analysis, which includes an overall complication rate $<19.6 \%$ and major bleeding event rate $<5.5 \%$. Outpatient 'day surgery' can be considered for low-risk patients undergoing native kidney biopsy from a health care savings perspective.

\section{Acknowledgements}

This study was supported by grants NIH T32 DK007569, NIH K24 DK062849 and NIH K23 DK080952 to Saugar Maripuri, T. Alp Ikizler and Kerri L. Cavanaugh, respectively.

\section{Disclosure Statement}

No author has any conflicts of interest to disclose. 


\section{References}

1 Parrish AE: Complications of percutaneous renal biopsy: a review of 37 years' experience. Clin Nephrol 1992;38:135-141.

2 Maya ID, Maddela P, Barker J, Allon M: Percutaneous renal biopsy: comparison of blind and real-time ultrasound-guided technique. Semin Dial 2007;20:355-358.

$\checkmark 3$ Alebiosu CO, Kadiri S: Percutaneous renal biopsy as an outpatient procedure. J Natl Med Assoc 2004;96:1215-1218.

-4 Bairy M, Beleed K, Webb AT, Bhandari S: Safety of outpatient kidney biopsy: one center's experience with 178 native kidney biopsies. Am J Kidney Dis 2008;52:631-632.

5 Maya ID, Allon M: Percutaneous renal biopsy: outpatient observation without hospitalization is safe. Semin Dial 2009;22:458-461.

6 Lin WC, Yang Y, Wen YK, Chang CC: Outpatient versus inpatient renal biopsy: a retrospective study. Clin Nephrol 2006;66:17-24.

$>7$ Margaryan A, Perazella MA, Mahnensmith RL, Abu-Alfa AK: Experience with outpatient computed tomographic-guided renal biopsy. Clin Nephrol 2010;74:440-445.
8 Fraser IR, Fairley KF: Renal biopsy as an outpatient procedure. Am J Kidney Dis 1995;25 876-878.

$\checkmark 9$ Simckes AM, Blowey DL, Gyves KM, Alon US: Success and safety of same-day kidney biopsy in children and adolescents. Pediatr Nephrol 2000;14:946-952.

10 Griner PF: Payment reform and the mission of academic medical centers. N Engl J Med 2010;363:1784-1786.

11 Birkmeyer JD, Liu JY: Decision analysis models: opening the black box. Surgery 2003;133:1-4.

12 Mendelssohn DC, Cole EH: Outcomes of percutaneous kidney biopsy, including those of solitary native kidneys. Am J Kidney Dis 1995;26:580-585.
13 Shidham GB, Siddiqi N, Beres JA, Logan B, Nagaraja HN, Shidham SG, Piering WF: Clinical risk factors associated with bleeding after native kidney biopsy. Nephrology (Carlton) 2005;10:305-310.

14 Stratta P, Canavese C, Marengo M, Mesiano $P$, et al: Risk management of renal biopsy: 1,387 cases over 30 years in a single centre. Eur J Clin Invest 2007;37:954-963.

15 Waldo B, Korbet SM, Freimanis MG, Lewis EJ: The value of post-biopsy ultrasound in predicting complications after percutaneous renal biopsy of native kidneys. Nephrol Dial Transplant 2009;24:2433-2439.

16 Whittier WL, Korbet SM: Timing of complications in percutaneous renal biopsy. J Am Soc Nephrol 2004;15:142-147.

-17 Ishikawa E, Nomura S, Hamaguchi T, Obe $\mathrm{T}$, et al: Ultrasonography as a predictor of overt bleeding after renal biopsy. Clin Exp Nephrol 2009;13:325-331.

18 Marwah DS, Korbet SM: Timing of complications in percutaneous renal biopsy: what is the optimal period of observation? Am J Kidney Dis 1996;28:47-52. 